Ann. Zootech., 1985, 34 (2), 229-236

\title{
Note
}

\section{Prévision de la digestibilité des fourrages destinés aux chevaux par dégradation enzymatique}

\author{
Nicoletta MIRAGLIA * et I.L. TISSERAND \\ Laboratoire de recherches de la Chaire de Zootechnie de l'E.N.S.S.A.A. \\ 26, bd Docteur-Petitjean, F 21100 Dijon
}

\begin{abstract}
Résumé
La digestibilité de 13 fourrages destinés aux chevaux est estimée à l'aide d'un test enzymatique. La méthode retenue est celle utilisant la pepsine cellulase et mise au point par J. Aufrere (1982) appliquée au calcul de la digestibilité de la matière organique.

Les résultats sont comparés aux valeurs de digestibilité in vivo des mêmes fourrages obtenues sur chevaux trotteurs par W. MartiN-Rosset (I.N.R.A. Theix) utilisées comme référence. Le degré de signification du coefficient de corrélation simple est toujours élevé, sauf entre la digestibilité in vivo de la cellulose brute et les digestibilités de la matière sèche et de la matière organique estimées par le test enzymatique.

En conclusion, comme chez les ruminants, le test à la pepsine-cellulase est une méthode assez précise pour estimer la digestibilité des fourrages destinés aux chevaux. Il permet d'évaluer la valeur des fourrages sans recourir à des techniques biologiques nécessitant la présence d'animaux fistulés.
\end{abstract}

Mots clés : chevaux, fourrages, digestibilité, pepsine-cellulase.

\section{Introduction}

La valeur alimentaire des fourrages dépend d'abord de leur digestibilité. Cette dernière peut être évaluée par :

a) des méthodes chimiques (LANCASTER, 1943 ; ForBES, 1950 ; RAYMOND, HARRIS \& BAKER, 1953 ; KiviMAE, 1969);

b) des techniques biologiques, tel que le test in vitro de TILley \& TerRY (1963) et la technique des sacs de nylon (van Keuren \& Heinemann, 1962 ; Applegate \& Hershberger, 1969 ; Demarouilly \& Chenost, 1969);

* Adresse actuelle : Instituto di Zootecnica, Alimentazione e Nutrizione. Facolta di Medicina Veterinaria. Via del Taglio, 43100 Parma (Italie). 
c) des techniques enzymatiques qui emploient, dans certaines conditions expérimentales, des enzymes et plus particulièrement une solution pepsine-cellulase (Lusk, Browning \&. Mrlis:s, 1962; Doluzien et al., 1963 ; Jones \& Hayward, 1975 ; MC QueEn \& VAN Soest, 1975 ; Allison \& Borzucki, 1978 ; MaC Leod \& Minson, 1978 ; Terry, Mundell \& Osbourn, 1978 ; Adamson \& Terry, 1980 ; Demarouilly \& JARrige, 1981 ; Aufrere, 1982).

- Les méthodes chimiques, très employées il y a une vingtaine d'années, ont tendance à être abandonnées car elles donnent des résultats dont la fiabilité est peu satisfaisante.

- Les méthodes biologiques, basées sur la reproduction in vitro de la digestion microbienne, sont fiables mais elles présentent l'inconvénient d'une mise en œuvre délicate due à la nécessité de disposer d'animaux fistulés.

- Les méthodes enzymatiques présentent certains avantages ; c'est, dans le cas des ruminants, un test rapide, reproductible et économique qui ne fait pas appel aux animaux. Il paraît, de ce fait, particulièrement intéressant aussi dans le cas des aliments destinés aux chevaux. C'est pourquoi, nous nous proposons d'étudier ses aptitudes à évaluer la digestibilité des fourrages chez cette espèce.

\section{Matériel et méthodes}

Treize fourrages couramment utilisés dans l'alimentation du cheval sont expérimentés : 5 bons foins de graminées, 4 foins de luzerne, 2 mauvais foins de graminées et 2 pailles dont la composition chimique est donnée dans le tableau 1. Les digestibilités in vivo de 10 de ces 13 fourrages sont mesurées sur des chevaux trotteurs par W. Martin-Rosset (I.N.R.A., Theix).

La méthode à la pepsine-cellulase proposée par J. Aufrere (1982) est utilisée. Elle comprend :

- un pré-traitement de $500 \mathrm{mg}$ de fourrage broyé (grille de $0,8 \mathrm{~mm}$ ) pesé dans des creusets filtrants (verre fritté de porosité 2) de $90 \mathrm{ml}$, d'une durée de 24 heures, au bain-marie à $40^{\circ} \mathrm{C}$ avec $50 \mathrm{ml}$ d'une solution de pepsine $(0,2 \mathrm{p} .100)$ dans l'acide chlorhydrique $1 \mathrm{~N}$,

- une filtration et un lavage à l'eau distillée,

- un traitement d'une durée de 24 heures, au bain-marie à $40^{\circ} \mathrm{C}$ avec $50 \mathrm{ml}$ d'une solution de cellulase extraite de Trichoderma viride (Onozuka $R$ 10),

- une filtration et un lavage à l'eau distillée,

- un séchage à l'étuve à $103^{\circ} \mathrm{C}$ durant $48 \mathrm{~h}$,

- une calcination au four à $550^{\circ} \mathrm{C}$ pendant $5 \mathrm{~h}$,

- le calcul du pourcentage de matière sèche (dM.S.) et de matière organique (dM.O.) solubilisées par les deux traitements.

L'analyse de chaque fourrage est répétée 5 fois, en double.

Les résultats obtenus sont comparés avec la digestibilité in vivo servant de référence. Les coefficients de corrélation simple sont calculés entre les variables (Fisher \& Yates, 1963). 


\section{Tableau 1}

Composition chimique des fourrages expérimentés.

Chemical composition of tested forages.

\begin{tabular}{|c|c|c|c|c|}
\hline \multirow[b]{2}{*}{$\begin{array}{l}\text { Fourrages } \\
\text { Forages }\end{array}$} & \multirow{2}{*}{$\begin{array}{c}\text { M.S. } \\
\% \text { brut } \\
D . M . \\
\% \text { brut }\end{array}$} & \multicolumn{3}{|c|}{$\%$ de M.S. (of D.M.) } \\
\hline & & $\begin{array}{l}\text { M.A.T. (1) } \\
\text { Crude } \\
\text { protein }\end{array}$ & $\begin{array}{l}\text { C.B. (2) } \\
\text { Crude } \\
\text { fibre }\end{array}$ & $\begin{array}{l}\text { Cendres } \\
\text { Ashes }\end{array}$ \\
\hline $\begin{array}{l}\text { Paille blé } \ldots \ldots \ldots \ldots \ldots \ldots \ldots \\
\text { Wheat straw }\end{array}$ & 95,7 & 4,0 & 41,6 & 9,6 \\
\hline $\begin{array}{l}\text { Paille orge } \ldots \ldots \ldots \ldots \ldots \ldots \ldots \\
\text { Barley straw }\end{array}$ & 95,0 & 3,1 & 44,2 & 7,1 \\
\hline $\begin{array}{l}\text { Mauvais foin } n^{\circ} 1 \ldots \ldots \ldots \ldots \\
\text { Bad hay } n^{\circ} 1\end{array}$ & 94,5 & 4,0 & 40,3 & 9,7 \\
\hline $\begin{array}{l}\text { Mauvais foin } \mathrm{n}^{\prime \prime} 2 \ldots \ldots \ldots \ldots \\
\text { Bat hay } n^{\circ} 2\end{array}$ & 93,5 & 5,2 & 37,8 & 9,7 \\
\hline $\begin{array}{l}\text { Foin de pré } \mathrm{n}^{\circ} 1 \text { (prairie naturelle) } \\
\text { Nat. mead. hay } n^{\prime \prime} l\end{array}$ & 94,2 & 8,8 & 32,3 & 7,6 \\
\hline $\begin{array}{l}\text { Foin de pré } \mathrm{n}^{\prime \prime} 2 \text { (pr. nat. } 2 \text { coupe) } \\
\text { Nat. mead. hay } 2 \text { nd cut } n^{\prime \prime} 2\end{array}$ & 94,1 & 9,2 & 35,2 & 7,4 \\
\hline $\begin{array}{l}\text { Foin de pré } \mathrm{n}^{\prime} 3 \text { (pr. nat. tardif) . } \\
\text { Nat. mead. hay late harv. } n^{\prime \prime} 3\end{array}$ & 94,8 & 6,3 & 36,8 & 6,1 \\
\hline $\begin{array}{l}\text { Foin de pré } n^{\prime \prime} 4 \text { (prairie naturelle) } \\
\text { Nat. mead. hay } n^{\prime \prime} 4\end{array}$ & 92,6 & 21,0 & 25,3 & 7,4 \\
\hline $\begin{array}{l}\text { Foin de pré n" } 5 \text { (pr. nat. } 2 \text { coupe) } \\
\text { Nat. mead. hay 2nd cut } n^{\circ} 5\end{array}$ & 91,3 & 21,2 & 30,0 & 12,7 \\
\hline $\begin{array}{l}\text { Foin de luzerne } \mathrm{n}^{\prime \prime} 1\left(\mathrm{l}^{\mathrm{re}} \text { coupe }\right) \\
\text { Lucerne hay } 1 \text { st cut } n^{\circ} 1\end{array}$ & 94,3 & 14,3 & 35,5 & 10,2 \\
\hline $\begin{array}{l}\text { Foin de luzerne } \mathrm{n}^{\prime \prime} 2\left(2^{\circ} \text { coupc }\right) \\
\text { Lucerne hay } 2 \text { d cut } n^{\circ} 2\end{array}$ & 93,6 & 17,0 & 35,3 & 11,5 \\
\hline $\begin{array}{l}\text { Foin de luzerne } \mathrm{n}^{\prime \prime} 3\left(3^{\mathrm{e}} \text { coupe }\right) \\
\text { Lucerne hay } 3 \text { rd cutt } n^{\prime \prime} 3\end{array}$ & 92,9 & 19,8 & 27,9 & 12,9 \\
\hline $\begin{array}{l}\text { Foin de luzerne } n^{\prime \prime} 4 \text { (ventilé) } \ldots \\
\text { Barn hay drying lacerne hay } n^{\circ} 4\end{array}$ & 91,0 & 16,6 & 32,9 & 8,1 \\
\hline
\end{tabular}

(1) Matières azotées totales : $\mathbf{N} \times 6,25$.

(2) Cellulose brute de Weende. 


\section{Résultats}

Les valeurs moyennes et les écarts types mesurés par la méthode enzymatique sont rassemblés dans le tableau 2.

\section{TABleau 2}

Digestibilité de la matière sèche, matière organique et cellulose brute Weende des 13 fourrages expérimentés par les essais « in vivo »t avec la technique pepsine-cellulase.

Dry matter, organic matter and cellulose Weende digestibilities of 13 forages measured by the digestion trials and by the pepsin-cellulase technique.

\begin{tabular}{|c|c|c|c|c|c|}
\hline \multirow{2}{*}{$\begin{array}{l}\text { Fourrages } \\
\text { Forages }\end{array}$} & \multicolumn{3}{|c|}{$\begin{array}{c}\text { Digestibilité in vivo p. } 100 \\
\text { Digestion trials \% }\end{array}$} & \multicolumn{2}{|c|}{$\begin{array}{l}\text { Digestibilité pepsine-cellullase } \\
\text { p. } 100 \\
\text { Digestion pepsin-cellulase } \%\end{array}$} \\
\hline & $\begin{array}{l}\text { M.S. } \\
D . M .\end{array}$ & $\begin{array}{l}\text { M.O. } \\
O . M .\end{array}$ & $\begin{array}{l}\text { C.B. } \\
\text { C.F. }\end{array}$ & $\begin{array}{c}\text { M.S. } \\
\overline{\mathrm{x}} \pm \sigma \\
D . M . \\
\bar{x}^{-} \pm \sigma\end{array}$ & $\begin{array}{l}\text { M.O. } \\
\overline{\mathrm{x}} \pm \sigma \\
O . M . \\
\bar{x} \pm \sigma\end{array}$ \\
\hline $\begin{array}{l}\text { Paille blé } \ldots . . . . \\
\text { Wheat straw }\end{array}$ & 35,0 & 37,7 & 38,3 & $33,7 \pm 1,41$ & $33,2 \pm 1,41$ \\
\hline $\begin{array}{l}\text { Paille orge } \ldots \ldots \ldots \ldots \\
\text { Barley Straw }\end{array}$ & 34,1 & 36,2 & 35,6 & $37,3 \pm 2,20$ & $37,0 \pm 1,80$ \\
\hline $\begin{array}{l}\text { Mauvais foin } 1 \ldots \ldots \ldots \\
\text { Bad hay } 1\end{array}$ & 37,5 & 37,9 & 30,3 & $43,2 \pm 2,24$ & $41,8 \pm 2,18$ \\
\hline $\begin{array}{l}\text { Mauvais foin } 2 \ldots \ldots \ldots \\
\text { Bad hay } 2\end{array}$ & 37,4 & 38,0 & 30,3 & $42,7 \pm 3,38$ & $37,2 \pm 11,59$ \\
\hline $\begin{array}{l}\text { Foin de pré } 1 \\
\text { Meadow hay } \\
1\end{array}$ & 49,3 & 50,4 & 39,9 & $57,4 \pm 1,50$ & $55,9 \pm 1,33$ \\
\hline $\begin{array}{l}\text { Foin de pré } 2 \\
\text { Meadow hay } \\
2\end{array}$ & - & - & - & $55,0 \pm 1,00$ & $54,2 \pm 1,33$ \\
\hline $\begin{array}{l}\text { Foin de pré } 3 \\
\text { Meadow hay } 3\end{array}$ & 一 & - & - & $55,8 \pm 0,65$ & $54,3 \pm 0,71$ \\
\hline $\begin{array}{l}\text { Foin de pré } 4 \\
\text { Meadow hay } 4\end{array}$ & 58,4 & 60,1 & 55,0 & $68,3 \pm 2,43$ & $67,6 \pm 2,19$ \\
\hline $\begin{array}{l}\text { Foin de pré } 5 \\
\text { Meadow hay } 5\end{array}$ & - & - & - & $76,1 \pm 0,77$ & $74,8 \pm 0,79$ \\
\hline $\begin{array}{l}\text { Foin de luzerne } 1 \\
\text { Lucerne hay } 1\end{array}$ & 54,8 & 56,6 & 35,3 & $57,0 \pm 1,10$ & $53,4 \pm 0,88$ \\
\hline $\begin{array}{l}\text { Foin de luzerne } 2 \quad \ldots \\
\text { Lucerne hay } 2\end{array}$ & 56,5 & 57,2 & 38,6 & $61,1 \pm 0,51$ & $56,9 \pm 0,57$ \\
\hline $\begin{array}{l}\text { Foin de luzerne } 3 \quad \ldots \\
\text { Lucerne hay } 3\end{array}$ & 58,8 & 58,6 & 40,0 & $65.9 \pm 0,80$ & $62,0 \pm 0,70$ \\
\hline $\begin{array}{l}\text { Foin de luzerne } 4 \\
\text { Lucerne hay } 4\end{array}$ & 63,8 & 63,0 & 40,6 & $63,3 \pm 1,01$ & $59.7 \pm 0.74$ \\
\hline
\end{tabular}




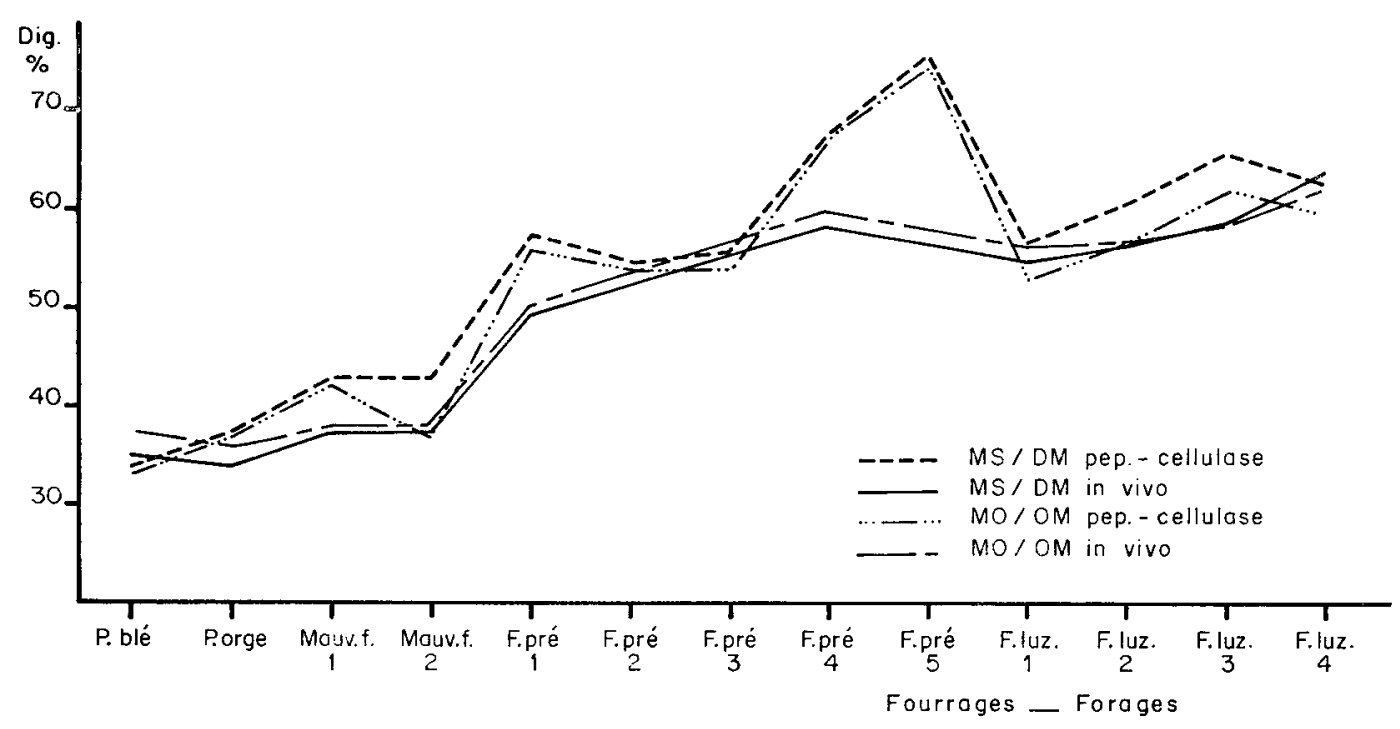

FIG. 1

Comparaison entre la digestibilité in vivo et la méthode pepsine-cellulase.

Comparison between in vivo digestion trials and pepsin-cellulase technique.

La dM.S. résultant de la dégradation enzymatique, est généralement légèrement plus élevée que celle mesurée in vivo. Dans le cas de la dM.O. seule, quelques valeurs apparaissent supérieures après la digestion pepsine-cellulase. Ces résultats sont confirmés par le calcul des coefficients de corrélation simple (tabl. 3).

\section{TABLEAU 3}

Coefficient de corrélation simple :

comparaison entre les essais in vivo et le test enzymatique $(n=10)$.

Simple coefficient of correlation: comparison between in vivo digestion trials and pepsin-cellulase technique $(n=10)$.

Digestibilité in vivo

Digestion trials

Matière sèche - Dry matter

Matière organique - Organic matter .............

Cellulose brute - Crude fibre

\begin{tabular}{|c|c}
$\begin{array}{c}\text { Pepsine-cellulase } \\
\text { Pepsin-cellulase }\end{array}$ \\
\hline M.S. & $\begin{array}{l}\text { M.O. } \\
O . M .\end{array}$ \\
\hline D.M. & \\
\hline & $0,941^{* *}$ \\
$0,960^{* * *}$ & $0,943^{* *}$ \\
$0,637^{*}$ & $0,714^{*}$ \\
\hline
\end{tabular}

* Significativité au $5 \%$. Significance at $5 \%$.
$*$ Significativité au $1 \%$. Significance at $1 \%$. 
Avec le test pepsine-cellulase, les pailles ont les valeurs les plus basses pour la digestibilité de la M.S. et de la M.O. Inversement, les valeurs les plus élevées

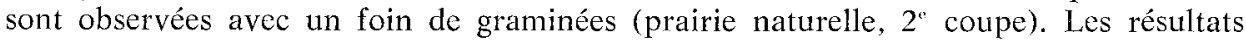
obtenus pour la digestibilité de la M.S. et de la M.O. de chaque fourrage sont très voisins. Toutefois alors qu'in vivo, la dM.O. est presque toujours supérieure à la dM.S., nous constatons l'inverse avec les dégradations enzymatiques.

Le graphique $\mathrm{n}^{\circ} 1$ illustrant la comparaison entre la digestibilité in vivo et la méthode pepsine-cellulase, montre une relation étroite entre les valeurs obtenues in vivo et par le test enzymatique.

La relation est très étroite $(\mathrm{P}>1 \mathrm{p}$. 100) entre les résultats obtenus in vivo et par la voie enzymatique en ce qui concerne la dM.S. et la dM.O.; elle est moins satisfaisante $(P>5$ p. 100) dans le cas de la digestibilité de la cellulose brute.

D'autre part, les liaisons entre la digestibilité in vivo de la cellulose brute et les digestibilités in vivo de la matière sèche et de la matière organique $(\mathbf{r}=0,586$ et 0,626 respectivement) sont faibles et non significatives. Elles pourraient expliquer les mauvaises corrélations observées avec le test enzymatique.

\section{Discussion et conclusion}

Le test enzymatique avec la pepsine-cellulase proposé par J. Aufrere (1982) pour prévoir la digestibilité du fourrage dans le cas des ruminants est applicable aussi dans le cas des chevaux. Cette méthode, ne nécessitant pas l'utilisation de sujets fistulés, est d'un emploi simple pour un laboratoire d'analyses de routine.

Les résultats que nous avons obtenus sont encourageants, ils démontrent la reproductibilité de la technique proposée.

Toutefois, l'analyse d'un grand nombre de fourrages devrait permettre à l'instar de ce qui existe déjà pour les ruminants, de calculer une équation de régression unique pour évaluer la digestibilité des fourrages chez les équidés avec une fiabilité satisfaisante.

Reçu en décembre 1984.

Accepté en mars 1985.

\section{Remerciements}

Nous tenons à remercier W. Martin-RosSET qui a mis à notre disposition les résultats de digestibilité mesurée in vivo sur trotteur. 


\section{Summary \\ An enzymatic procedure for estimating horse forage digestibility}

Digestibility of 13 horse forages (tabl. 1) was estimated by an cnzymatic test. The pepsin-cellulase technique developed by J. AUfrere (1982) was used to measure dry matter and organic matter digestibility. Results were compared to the in vivo digestibility values obtained with the same forages in trotters by W. MARTIN-Rosset (I.N.R.A. Theix) used as controls (tabl. 2).

A significant coefficient of correlation between variables was always obtained, except for the comparison with in vivo crude fibre digestibility (tabl. 3).

It may be concluded that, as already shown in ruminants, the enzymatic procedure is accurate enough to estimate forage digestibility (graph. 1). It allows to evaluate the feeding value of forages without using the biological techniques needing the use of fistulated animals.

Key words : horses, forages, digestibility, pepsin-cellulase.

\section{Références bibliographiques}

Adamson A.H., Tirry G.R., 1980. The relationship between the in vivo digestibility of hay and its solubility in pepsin-hydrochloric acid and fungal cellulase solutions. J. Sci. Food Agric., 31, 854-856.

Allison M., Borzucki R., 1978. Cellulase method for the efficient digestion of grasses and brassicas. J. Sci. Food Agric., 29, 293-297.

Applegate C.S., Hershrerger T.V., 1969. Evaluation of in vitro and in vivo cecal fermentation techniques for estimating the nutritive value of forages for equine. J. Anim. Sci., 28, 18-22.

Aufrere J., 1982. Etude de la prévision de la digestibilité des fourrages par une méthode enzymatique. Ann. Zootech., 31, 111-130.

Demarquilly C., Chenost M., 1969. Etude de la digestion des fourrages dans le rumen par la méthode des sachets de nylon; liaison avec la valeur alimentaire. Ann. Zootech., 18, 419-436.

Demarquilly C., Jarrige R., 1981. Panorama des méthodes de prévision de la digestibilité et de la valeur énergétique des fourrages. XI" journées du Grenier de Teix, in : Prévision de la valeur nutritive des aliments des ruminants, I.N.R.A. Ed., 41-59.

Donefir E., Neimann P.J., Crampton E.W., Llyod L.E., 1963. Dry matter disappearance by enzyme and aqueous solutions to predict the nutritive value of forages. J. Dairy Sci., 46, $965-970$.

Fisher R.A., Yatis F., 1963. Statistical tables. Oliver and Boyd Ltd. Ed., Edimburgh, $146 \mathrm{p}$.

Forbes R.M., 1950. Protein as an indicator of pasture forage digestibility. J. Anim. Sci., 9, 231-237.

Jones D.I.H., HaYward M.V., 1975. The effect of pepsin pretreatment of herbage on the prediction of dry matter digestibility from solubility in fungal cellulase solutions. J. Sci. Food Agric., 26, 711-718.

KivimaE A., 1960. Estimation of the digestibility of grassland crops from their chemical composition. Proc. 8th. Int. Grassl. Congr., pp. 466-470.

LANCASTER R.J., 1943. Metabolism trials with New Zealand feeding stuffs. IV. The relative significance of lignin, cellulose and crude fibre in the evaluation of foods. N.Z. J. Sci. Tech., 25A, 137-151.

Lusk J.W., Browning C.B., Miles J.T., 1962. Small sample in vivo cellulase digestion procedure for forage evaluation. J. Dairy Sci., 45, 69-73. 
MC Leod M.N., Minson D.J., 1978. The accuracy of the pepsin-cellulase technique for estimating the dry matter digestibility in vivo of grasses and legumes. Anim. Feed. Sci. Technol., 3, 277-287.

Mc Queen R., VAN Soest P.J., 1975. Fungal cellulase and hemicellulase prediction of forage digestibility. J. Dairy Sci., 58, 1482-1491.

Raymond W.F., Harris C.E., BaKer V.G., 1953. Studies on the digestibility of herbage. I. Technique of measurement of digestibility and some observations on factors affecting the accuracy of digestibility data. J. br. Grassl. Soc., 8, 301-320.

Terry R.A., Mundell D.C., Osbourn D.F., 1978. Comparison of two in vitro procedures using rumen liquor-pepsine or pepsine-cellulase for prediction of forage digestibility. J. br. Grassl. Soc., 33, 13-18.

Tilley J.M.A., Terry R.A., 1963. A two stage technique for in vitro digestion of forage crops. J. br. Grassl. Soc., 18, 104-111.

Van Keuren R.W., Heinemann W.W., 1962. Study of a nylon bag technique for in vivo estimation of forage digestibility. J. Anim. Sci., 21, 340-345. 